Supplement of Earth Syst. Dynam., 12, 783-795, 2021

https://doi.org/10.5194/esd-12-783-2021-supplement

(C) Author(s) 2021. CC BY 4.0 License.

(c) (i)

Supplement of

\title{
Modelling sea-level fingerprints of glaciated regions with low mantle viscosity
}

\author{
Alan Bartholet et al. \\ Correspondence to: Glenn A. Milne (gamilne@uottawa.ca)
}

The copyright of individual parts of the supplement might differ from the article licence. 

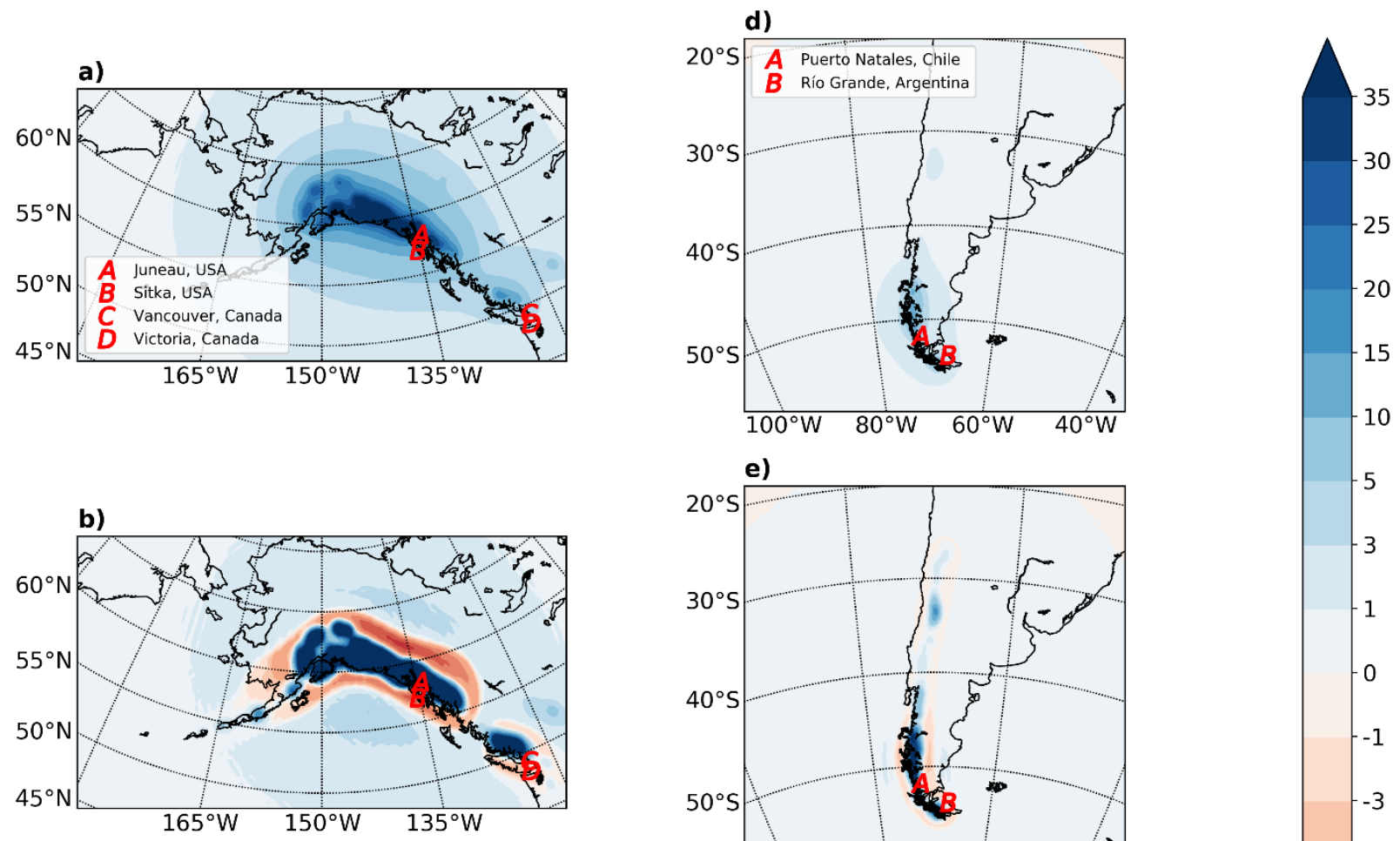

e)
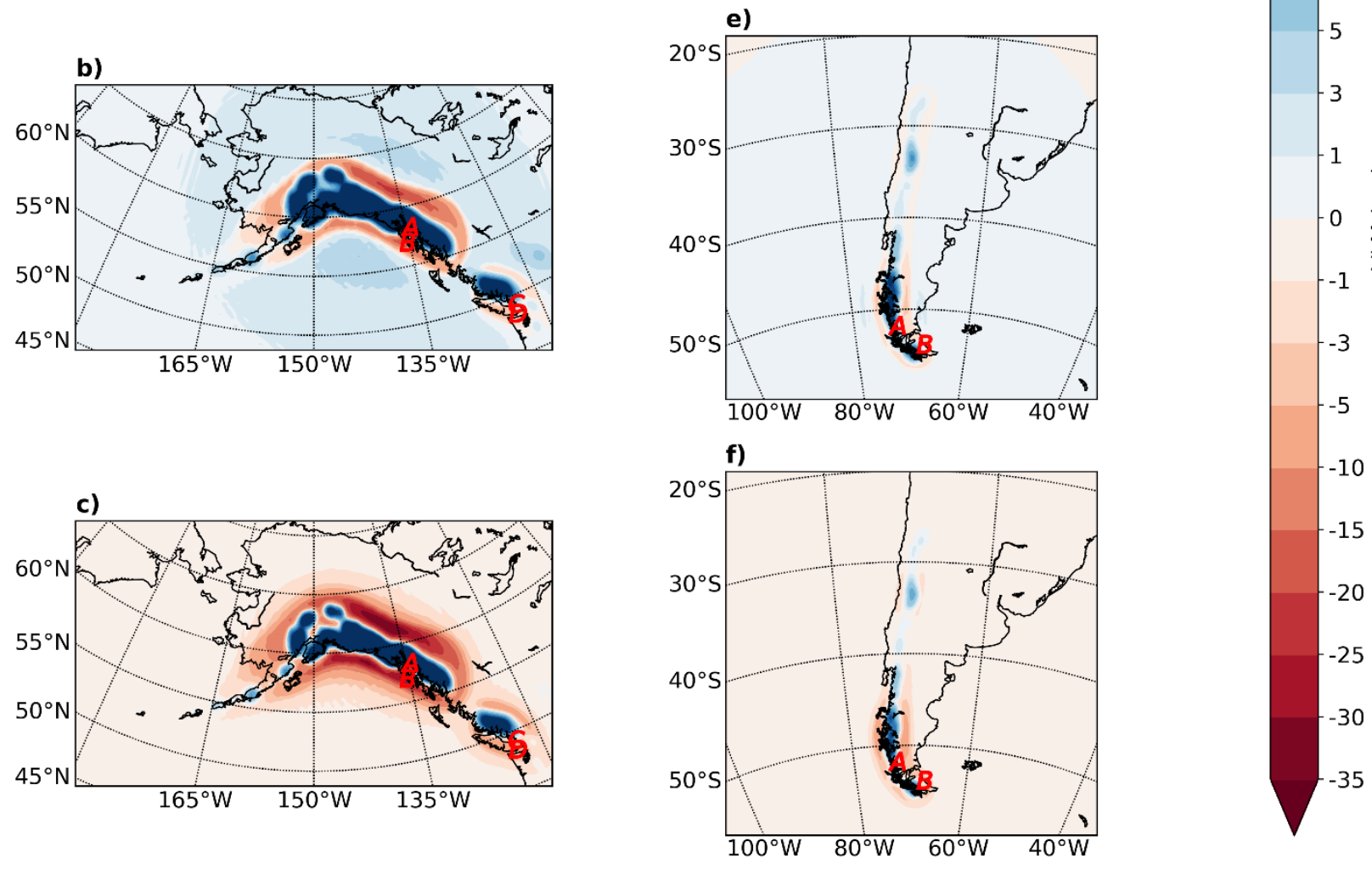

Figure S1. Calculated vertical land motion (VLM) for estimated changes in regional glacier distributions for RGI regions $1 \& 2$ (left) and region 17 (right). The different frames show results for: (a \& d) a 1-D (spherically symmetric) elastic Earth model; (b \& e), a 3-D viscoelastic Earth model with low viscosity regions located as indicated in Fig. 2. The results in (c) and (f) show the differences between the viscoelastic and elastic results, respectively (i.e. (b) minus (a) and (e) minus (d)). Note that these results do not include the sea-level signal associated with ice mass changes from outside of the RGI regions shown. The locations of population centres for which relative sea-level curves are calculated are indicated by the red letters. 

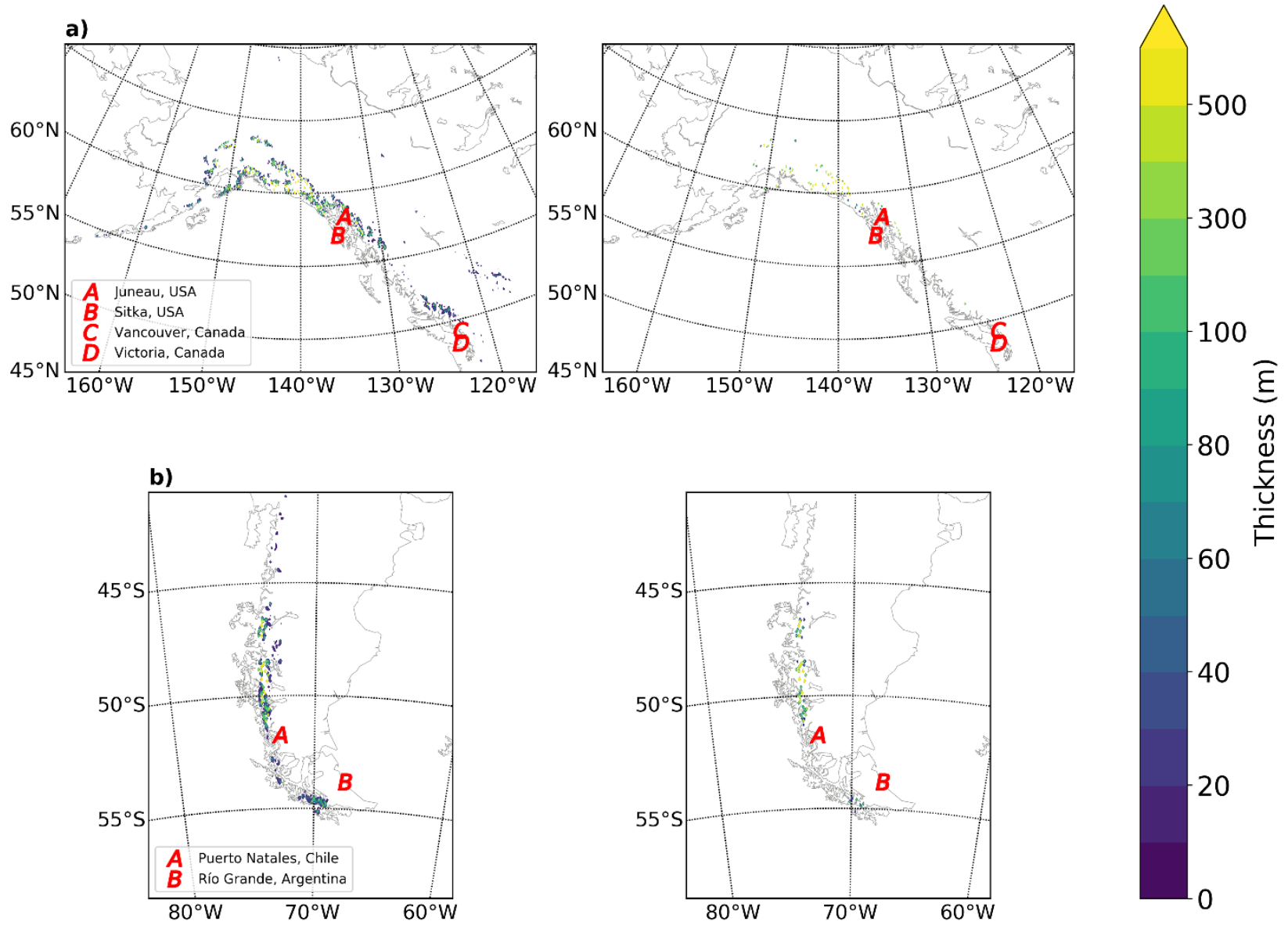

60

$-40$

20

Figure S2. Estimated spatial distribution in ice thickness in RGI regions 1 \& 2 (a) and 17 (b) at the beginning (2010 $\mathrm{CE}$, left) and end (2100 CE, right) of the time period considered. These ice distribution models were determined in the same way as those shown in Fig. 1 except that no spatial smoothing was applied. As a result, the lateral extent of the ice is reduced but the thickness changes are greater to produce the same changes in ice volume. The locations of population centres for which relative sea-level curves are calculated are indicated by the red letters. 

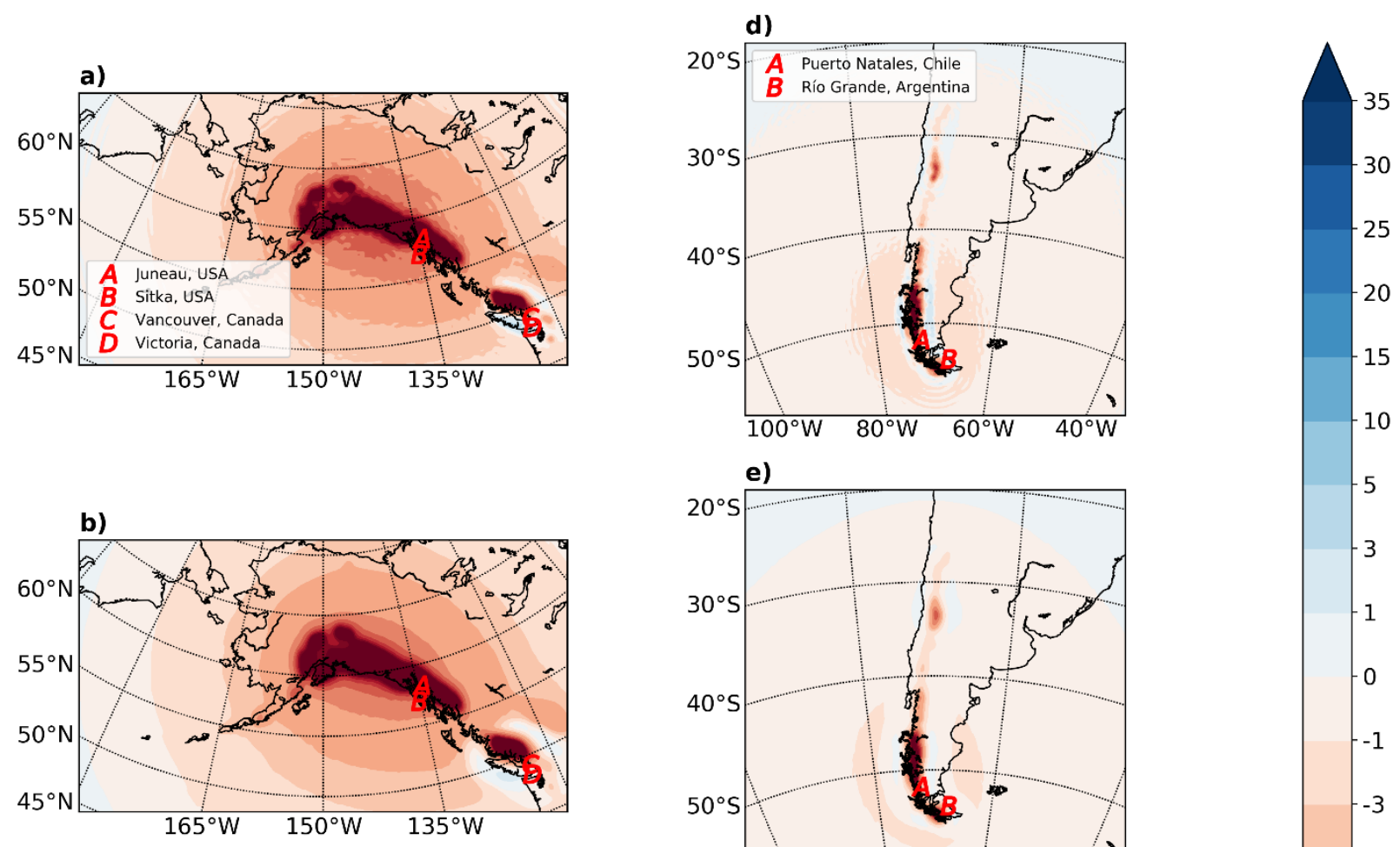

e)
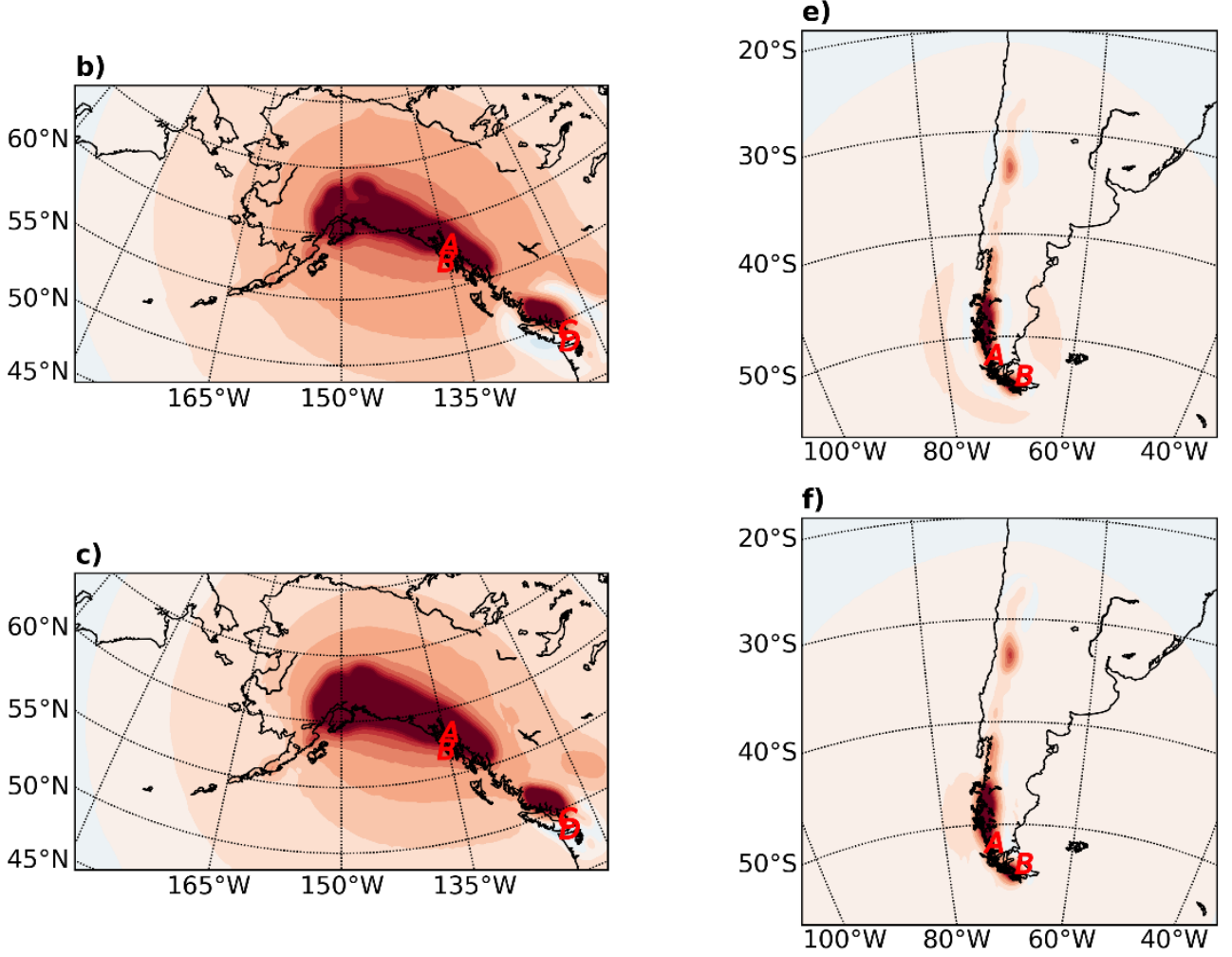

Figure S3. Modelled RSL changes for the period 2010-2100 CE when key input parameters are changed relative to the model parameters used to calculate the results in Figs $4 \& 5$. Frames a-c show results for RGI regions 1 and 2 when the ice model is not spatially smoothed (a; corresponding to results labelled 'no smoothing' in Fig. 6), the lateral extent of the low viscosity regions are extended (b; corresponding to results labelled 'rev1' in Fig. 6), the depth extent of the low viscosity regions are extended (c; corresponding to results labelled 'rev2' in Fig. 6). Frames d-e show the same as the results in a-c but for RGI region 17. The locations of population centres for which relative sea-level curves are calculated are indicated by the red letters. 

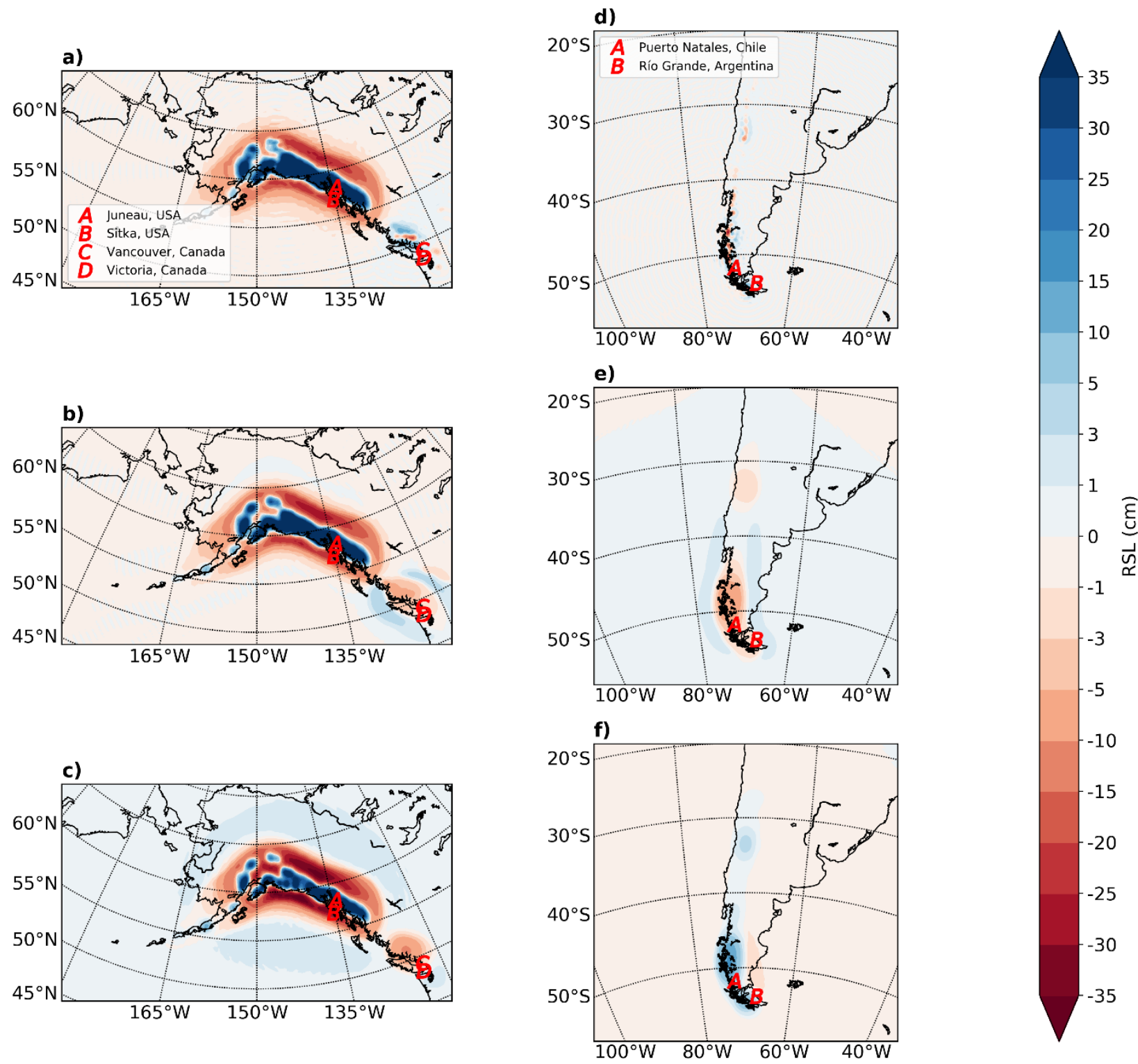

Figure S4. Differences in the modelled RSL changes shown in Fig. S3 relative to those shown for the 3-D viscoelastic Earth model in Fig. 4. Specifically, the results in frames a-c show the results in Fig. S3 (a-c) minus those in Fig. 4 (b) and the results in frames d-f show the results in Fig. S3 (d-f) minus those in Fig. 4 (e). The locations of population centres for which relative sea-level curves are calculated are indicated by the red letters. 

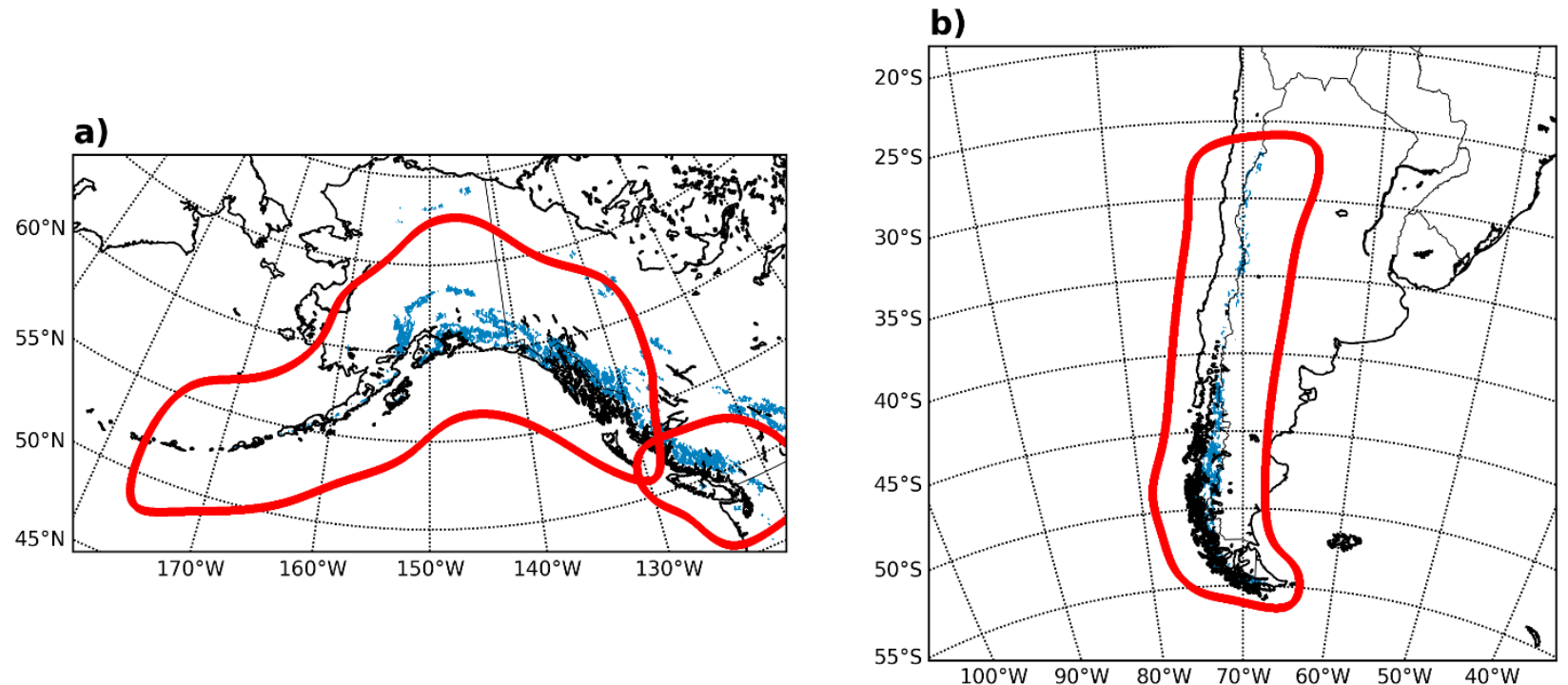

Figure S5. Surface lateral extent of the regions for which the underlying Earth structure (lithospheric thickness and sub-lithosphere viscosity profile) deviates from the adopted global values for model run in which the lateral extent of these areas was increased compared to the standard model experiments (see Fig. 2). Note that, in the volume of overlap between the low viscosity regions beneath RGI regions $1 \& 2$, the viscosity values were averaged. 

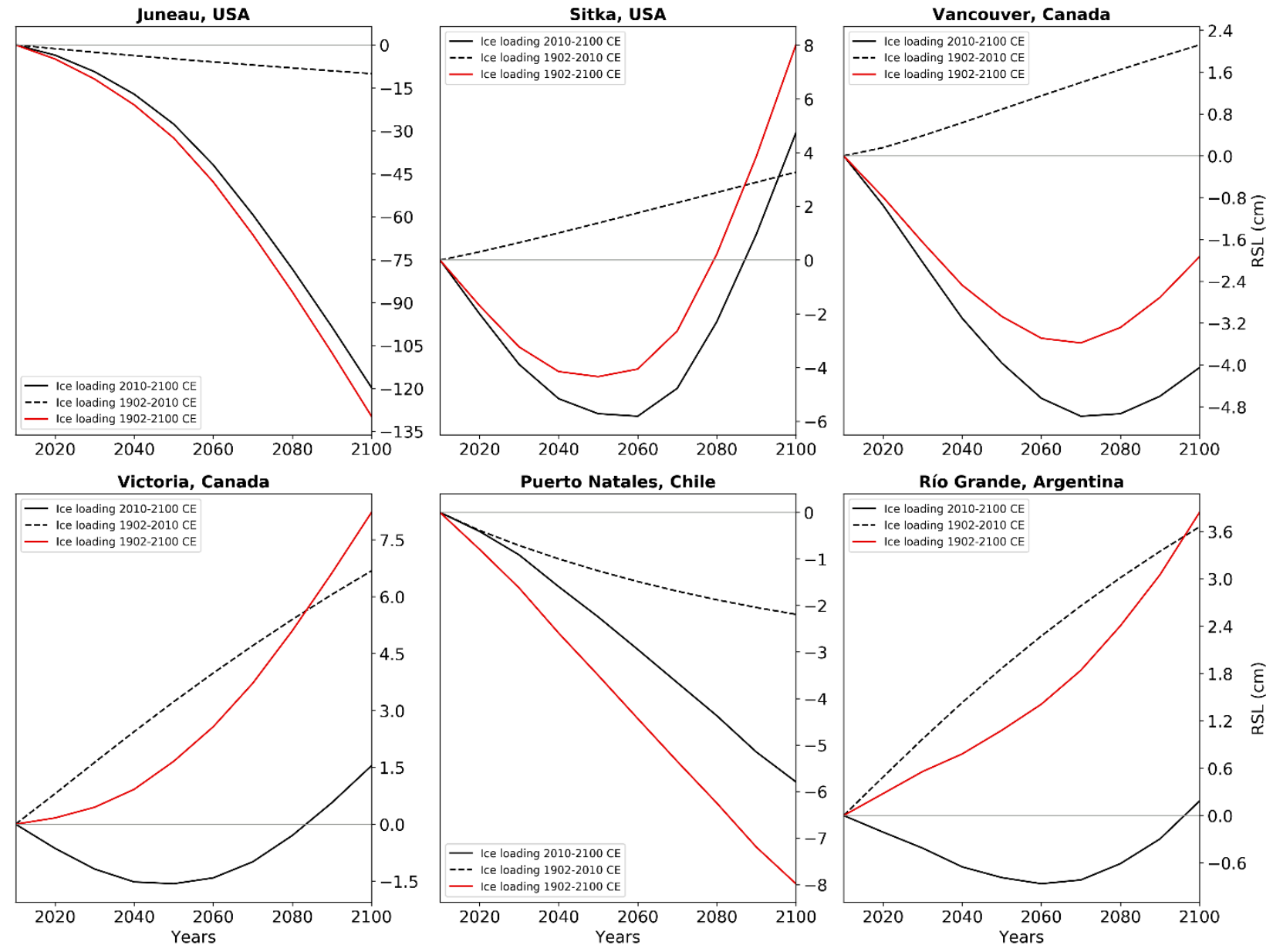

Figure S6. Modelled RSL curves over the period 2010-2100 CE due to ice loading during this period (solid black lines; same as in Fig. 5) and ice loading during 1902-2010 CE (dashed black lines). The sum of the two ice loading signals is shown by the red lines. 

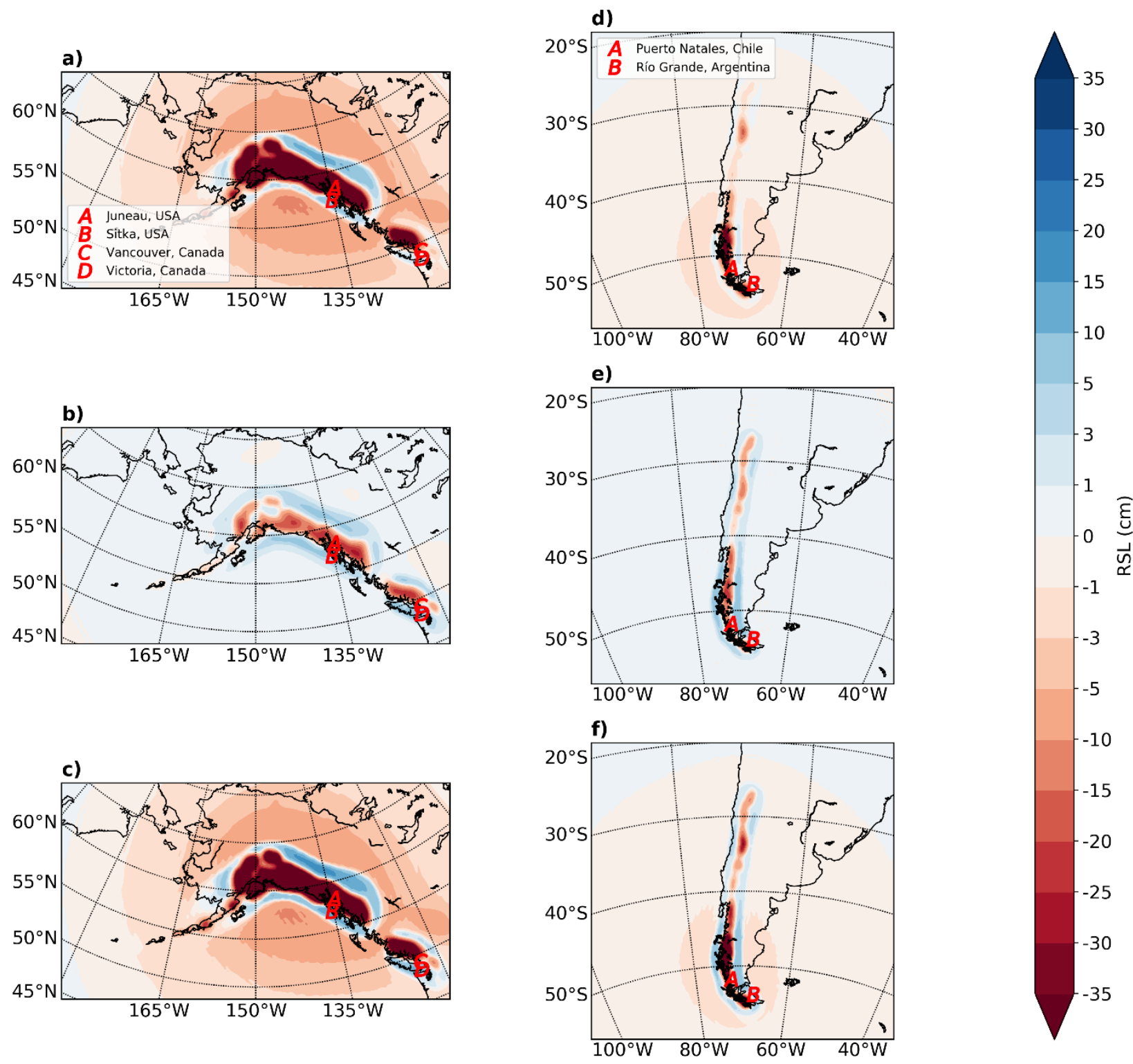

Figure S7. Modelled RSL changes in the RGI regions considered over the period 2010-2100 CE due to ice loading during this period (a \& d) and ice loading from 1902 to 2010 CE (b \& e). The sum of these two loading signals is shown in frames $c(a+b)$ and $f(d+e)$. The locations of population centres for which relative sea-level curves are calculated are indicated by the red letters. 